\title{
Effect of intravenous thrombolysis combined with mild hypothermia on the levels of IL-1ß, IL-6, ICAM-1 and MMP-2 in patients with acute cerebral infarction and clinical significance
}

\author{
CHAOSHENG LI ${ }^{1}$, LINGLING HU ${ }^{1}$, JILAI ZHAO ${ }^{1}$, MEIQI DI ${ }^{1}$, \\ CHANGYAN FAN $^{1}$, LIKUN HAN ${ }^{1}$ and XUYING ZHU ${ }^{2}$ \\ ${ }^{1}$ Department of Neurology, Wuxi Third People's Hospital, Wuxi, Jiangsu 214000; ${ }^{2}$ Department of Neurology, \\ Shuguang Hospital Affiliated to Shanghai University of Traditional Chinese Medicine, Shanghai 201203, P.R. China
}

Received May 31, 2019; Accepted October 30, 2019

DOI: $10.3892 /$ etm.2022.11147

\begin{abstract}
The present study aimed to explore the effects and clinical importance of serum interleukin (IL) IL-1 $\beta$, IL-6, C-reactive protein (CRP), intercellular adhesion molecule (ICAM)-1 and matrix metalloproteinase (MMP)-2 in patients with acute cerebral infarction undergoing intravenous thrombolysis during simultaneous hypothermia therapy. A total of 80 patients with acute cerebral infarction who were treated at our hospital were randomly selected. They were divided into groups A and B. The two groups were treated with intravenous thrombolysis, while group B received sub-hypothermia treatment. Prior to treatment and at 7 days after treatment, $5 \mathrm{ml}$ of venous blood was collected and stored in a freezer at $-80^{\circ} \mathrm{C}$. IL-1 $\beta$, IL-6, CRP, ICAM-1 and MMP-2 levels were detected by ELISA and compared between the groups and time-points. The results were as follows: i) At 7 days after treatment, the levels of IL-1 $\beta$, IL-6, CRP, ICAM-1 and MMP-2 in group B were significantly decreased compared with those in group $\mathrm{A}$ $(\mathrm{P}<0.05)$, while there was no significant difference of these levels between group A and B before treatment $(\mathrm{P}>0.05)$. The incidence of adverse reactions in group A and group B was 35 and $20 \%$ respectively, and the mortality rate was 10 and $5 \%$, respectively. There were no significant differences in adverse events and mortality between the two groups $(\mathrm{P}>0.05)$. In addition, a positive correlation of the level of IL-1 $\beta$, IL- 6 , CRP, ICAM-1 and MMP-2 with the National Institutes of Health Stroke Scale score was determined in the patients prior to treatment. In conclusion, mild hypothermia treatment in addition to intravenous thrombolysis significantly reduced the
\end{abstract}

Correspondence to: Dr Xuying Zhu, Department of Neurology, Shuguang Hospital Affiliated to Shanghai University of Traditional Chinese Medicine, 528 Zhang Heng Road, Pudong New Area, Shanghai 201203, P.R. China

E-mail: xuyingzhu4em@163.com

Key words: intravenous thrombolysis, mild hypothermia treatment, acute cerebral infarction, inflammatory reaction levels of IL-1 $\beta$, IL-6, CRP, ICAM-1 and MMP-2 in patients with acute cerebral infarction and reduced inflammation, and should therefore be incorporated in clinical practice.

\section{Introduction}

With the changes in diet, lifestyle diversification and the aging of the population, the incidence of acute cerebral infarction and the associated mortality rate are increasing, placing heavy burdens on patients and their families (1). Acute cerebral infarction is the most common cause of stroke and mainly occurs due to narrowing and even occlusion of blood vessels in the brain. Once the supply of blood flow suddenly stops, ischemia and hypoxia occur in the brain, resulting in brain tissue softening and edema, which promotes increased intracranial pressure and aggravates clinical symptoms, including impaired neurological function $(2,3)$. Once the condition arises, effective treatment methods are limited. The most reliable method for treating acute cerebral infarction is intravenous thrombolysis in the early stage, which may reduce the narrow area of the occluded blood vessels, increase the blood supply in the infarct area, shorten the cerebral ischemia time and reduce brain tissue damage. Recombinant human tissue plasminogen activator (rt-PA) has been widely used for intravenous thrombolysis within $4.5 \mathrm{~h}$ after the onset of acute cerebral infarction $(4,5)$. However, the time window for thrombolytic therapy is short and the therapeutic effect has not been fully evaluated (6).

In recent years, only a small number of patients with acute cerebral infarction have been able to receive thrombolytic therapy in the optimal time window for thrombolysis. Most patients miss the optimal thrombolysis time, resulting in poor thrombolytic therapy. Therefore, application of brain protection approaches during treatment is of great significance for treating acute cerebral infarction. Associated studies have indicated that physical methods enable patients to be in a mild hypothermia state, reduce brain edema and intracranial pressure, slow down brain tissue metabolism and avoid ischemia-reperfusion injury after intravenous thrombolysis (7-9), thus promoting neurological function recovery and improving prognosis (10). Acute cerebral infarction is a process of 
lipid accumulation and chronic inflammation characterized by the reaction of cytokines and inflammatory factors and consequently, assessment of inflammatory factors in the acute cerebral infarction has attracted increasing interest in recent decades. However, whether the inflammatory factors interleukin (IL)-1, IL-6, intercellular adhesion molecule (ICAM)-1 and matrix metalloproteinase (MMP)-2 have a role in patients with acute cerebral infarction undergoing intravenous thrombolysis has remained elusive. The present study aimed to investigate the role of IL-1 $\beta$, IL-6, C-reactive protein (CRP), ICAM-1 and MMP-2 in patients with acute cerebral infarction by measuring their levels prior to and after treatment. The clinical application value of intravenous thrombolysis combined with mild hypothermia in patients with acute cerebral infarction was also explored.

\section{Materials and methods}

Research objects and grouping. With the approval of the Medical Ethics Committee of Wuxi Third People's Hospital (Wuxi, Jiangsu, China), 80 patients with acute cerebral infarction diagnosed at Wuxi Third People's Hospital (Wuxi, Jiangsu, China) between January 2016 and December 2017 and were selected as the subjects. The inclusion criteria were as follows: i) According to the 'China Acute Ischemic Stroke Diagnosis and Treatment Guide 2014 (11), the patients were diagnosed with acute cerebral infarction; ii) the onset time was within $6 \mathrm{~h}$; iii) any neurological symptoms were consistent with the diagnosis of acute cerebral infarction; iv) blood pressure of $\leq 180 / 100 \mathrm{mmHg}$; v) CT and MRI imaging indications for acute cerebral infarction. The following exclusion criteria were applied: i) Venous thrombosis in the limbs; ii) cerebral infarction due to non-angiopathy; iii) heart, liver or kidney failure; iv) history of cerebral infarction or myocardial infarction; v) pregnant or lactating females; vi) subjects participating in other associated medical experimental research or who are unable/unwilling to cooperate with the completion of treatment. The cohort included 37 females and 43 males, aged $42-80$ years (average age, $44.5 \pm 15.7$ years). The 80 patients were randomly and equally divided into the conventional treatment group (group A) and the hypothermia treatment group (group B). All enrolled patients and their families provided written informed consent to participate in the present study.

Treatments. The enrolled patients maintained blood glucose and blood pressure within the normal range after hospitalization with improved circulation. The two groups were treated with rt-PA intravenous thrombolysis and intravenously given rt-PA $0.9 \mathrm{mg} / \mathrm{kg}$ (maximum dose, $90 \mathrm{mg}$ ). The rt-PA used was all of the same batch, purchased from Boehringer Ingelheim International. Of the total amount, $10 \%$ was intravenously injected within $1 \mathrm{~min}$ and the remaining amount was intravenously instilled for $1 \mathrm{~h}$. The patients' condition was closely monitored within $24 \mathrm{~h}$ after treatment. The major monitoring measures during intravenous thrombolytic therapy included the following: i) The patient was admitted to the intensive care unit for 24-h dynamic ECG monitoring. ii) After the first thrombolysis, the blood pressure was monitored every $15 \mathrm{~min}$ for the first $2 \mathrm{~h}$, followed by every $30 \mathrm{~min}$ for the next $6 \mathrm{~h}$, and then once per $\mathrm{h}$ until a total of $24 \mathrm{~h}$ had passed. If the patient had a diastolic blood pressure $>100 \mathrm{mmHg}$ and/or systolic blood pressure of $180 \mathrm{mmHg}$, anti-hypertensive drugs were administrated and blood pressure was closely monitored. iii) According to the patient's requirement for neurological assessment, this was performed 1 time/h. iv) If the blood pressure was severely high with hiccup or headache, thrombolytic therapy was immediately stopped and head CT examination was performed. In group B, intravenous thrombolytic therapy was performed simultaneously with mild hypothermia. The patient was made to wear a cooling cap, the head was wrapped and the desuperheater (Kadant Inc.) was started. The temperature of the cooling cap was maintained at $6-12^{\circ} \mathrm{C}$, the patient's eardrum temperature was maintained at $33-35^{\circ} \mathrm{C}$ and the treatment time was 6 days. During the hypothermia phase, the patient's vital signs and electrolytes were closely monitored. After the treatment with mild hypothermia, the warming cap was re-warmed on time, i.e., the patient's body temperature was increased by $1^{\circ} \mathrm{C} / 5 \mathrm{~h}$ and the patient's eardrum temperature was restored to $36.5-37.5^{\circ} \mathrm{C}$ after $\sim 20 \mathrm{~h}$. The desuperheater, cooling cap and infrared thermometer used by the patient were the same.

In the two groups, $5 \mathrm{ml}$ fasting venous blood was collected prior to treatment and at 7 days after treatment in an anti-coagulation tube that was placed in a low-temperature ultracentrifuge and centrifuged at 2,000 x g for $10 \mathrm{~min}$ at $4^{\circ} \mathrm{C}$ to collect the supernatant, which was stored at $-80^{\circ} \mathrm{C}$ for further analysis.

Observation indicators. Serum IL-1 $\beta$, IL-6, CRP, ICAM-1 and MMP-2 levels were determined in the two groups using an ELISA kit. The kits used were all in the same production batch and purchased from Biyuntian Biotechnology Co., Ltd. All testing procedures were determined in strict accordance with the instrument and reagent instructions to ensure the reliability of the test data.

Evaluation of efficacy. At 7 days after treatment, the clinical treatment effect was evaluated according to the diagnostic criteria for cerebrovascular disease and the National Institutes of Health Stroke Scale (NIHSS) score of cerebral infarction was determined according to the functional score classification criteria as determined at the 4th Cerebrovascular Conference (10). The treatment efficacy was rated as follows: i) Not improved: NIHSS score $<17 \%$; ii) improved: NIHSS score of $18-45 \%$; iii) significantly improved: NIHSS score of $46-90 \%$; iv) almost cured: NIHSS score of 91-100\%. The total efficiency was calculated as follows: Total efficiency $=($ almost cured + significantly improved + improved)/total number $\mathrm{x} 100 \%$.

Safety assessment. Pulmonary inflammation, extracranial hemorrhage, intracranial hemorrhage and mortality were recorded during treatment and 7 days after treatment. Pulmonary inflammation was assessed by ${ }^{18} \mathrm{~F}$-fludeoxyglucose positron emission topography. Intracranial hemorrhage was assessed according to the score and diagnostic criteria of acute stroke (12). Intracranial hemorrhage may be symptomatic or asymptomatic. Symptomatic intracranial hemorrhage refers to an increase in NIHSS score of $>4$ points or death in patients with acute cerebral infarction within $36 \mathrm{~h}$ of thrombolytic therapy (13). 
Statistical analysis. Data were processed using SPSS 19.0 statistical software (IBM Corp.). Values are expressed as the mean \pm standard deviation. The unpaired t-test was used for comparison within the group and an independent-samples t-test was used for comparison between groups. Count data were presented as a ratio or composition ratio and a $\chi^{2}$ test was used for comparison between groups. Pearson correlation analysis was performed to assess correlations. $\mathrm{P}<0.05$ was considered to indicate a statistically significant difference.

\section{Results}

General information. The basic information of the patients is provided in Table I. Parameters including age and body weight were not significantly different between the two groups ( $\mathrm{P}>0.05)$.

Serum levels of IL-1 $\beta, I L-6, C R P, I C A M-1$ and MMP-2 in the two groups of patients. Compared with those in group $\mathrm{A}$, the levels of IL-1 $\beta$, IL- 6 and CRP in group B were decreased at 7 days after treatment and the differences were statistically significant $(\mathrm{P}<0.05$; Table II, Fig. 1A-C). However, there were no differences in white blood cells prior to and after treatment in the two groups (Fig. 1D).

Compared with those in conventional treatment group (group A) and the hypothermia treatment group (group B), the levels of ICAM-1 and MMP-2 in group B were significantly decreased at 7 days after treatment $(\mathrm{P}<0.05)$, while there was no significant difference between group $\mathrm{A}$ and $\mathrm{B}$ before treatment ( $>0.05$; Table II, Fig. 2A-D).

Evaluation of effectiveness. At 7 days after treatment, the total effective rate was $80 \%$ in group A and $95 \%$ in group B. There was a significant difference between the two groups $(\mathrm{P}<0.05 ;$ Fig. 3).

Safety evaluation. The incidence of adverse reactions in group A and group B was 35 and $20 \%$, respectively, and the mortality rates were 10 and 5\%, respectively. There were no significant differences in adverse events and mortality between the two groups ( $\mathrm{P}>0.05$, Table III).

Correlation analysis of $I L-1 \beta, I L-6, C R P, I C A M-1$ and $M M P-2$ with neurological function. To evaluate whether there is an association of IL-1 $\beta$, IL-6, CRP, ICAM- 1 or MMP-2 with neurological function, a correlation analysis was performed in all patients prior to treatment, revealing a positive correlation of the level of IL-1 $\beta$, IL-6, CRP, ICAM-1 and MMP- 2 with the NIHSS score (Table IV).

\section{Discussion}

Acute cerebral infarction is one of the most common cerebrovascular diseases in neurology and is common among middle-aged and elderly individuals. The annual incidence rate in China is as high as $120 / 100,000-180 / 100,000$, with continuously increasing mortality rate (14). Acute cerebral infarction is a process resulting from lipid accumulation and chronic inflammation characterized by the reaction of cytokines and inflammatory factors. One of the major causes of cerebral ischemia-reperfusion injury is brain inflammation
Table I. General information of the two groups of patients $(\mathrm{n}=40$ per group).

\begin{tabular}{lccc} 
Group & Sex (male/female) & Age (years) & Body weight $(\mathrm{kg})$ \\
\hline A & $22 / 18$ & $44.2 \pm 15.6$ & $51.6 \pm 12.7$ \\
B & $21 / 19$ & $41.2 \pm 16.4$ & $56.3 \pm 18.2$ \\
P-value & 0.084 & 0.135 & 0.116 \\
\hline
\end{tabular}

caused by cerebral infarction $(15,16)$. The core of the treatment approach for acute cerebral infarction is to restore the blood circulation as soon as possible, correct brain ischemia and hypoxia, reduce inflammation and cerebral edema, reduce intracranial pressure, and thus ameliorate the degree of brain damage. However, due to intravenous thrombolytic therapy, the incidence of intracranial hemorrhage may be as high as $10.9-32.0 \%$ and the mortality rate after bleeding is high. The comorbidities are unacceptably high, resulting in an unsatisfactory curative effect and poor prognosis. The treatment of acute cerebral infarction remains challenging and has become a hot topic in clinical research. An appropriate therapeutic strategy should not only resolve cerebral ischemia and hypoxia, but also protect brain tissue function and improve the prognosis.

MMP-2 is one of the key members of the MMPs and acts as an inflammatory molecule in atherosclerotic plaques of brain tissues, which contributes to the damage of the blood-brain barrier after brain tissue ischemia and increases the permeability of the blood-brain barrier (17), causing brain edema. Relevant studies suggested that IL-1 $\beta$, IL- 6 and MMP-2 may be used as indicators for the detection of the condition and prognosis of patients with acute cerebral infarction, and may accurately reflect the condition and treatment effect of patients with acute cerebral infarction $(18,19)$. IL-1 $\beta$ is a cytokine that has multiple biological effects. Studies have indicated that when brain tissue is subjected to ischemia, the IL-1 $\beta$ content increases rapidly, promotes brain cell apoptosis, accelerates free radical release, induces endothelial cells to secrete pro=coagulant factors, inhibits anti-coagulant activity and reduces cerebral blood flow $(20,21)$. IL-6 is an immunological and inflammatory cytokine that is used as a major risk indicator for detecting ischemic brain injury (22) and is associated with an inflammatory response to acute cerebral infarction. ICAM-1 is one of the largest adhesion molecules that mediate adhesion reactions and belongs to the immunoglobulin superfamily, which enhances the adhesion of the inflammatory factors.

In recent years, sub-hypothermia in the range of $33-35^{\circ} \mathrm{C}$ has been reported to reduce the oxygen consumption of brain tissue, slow down the metabolism of brain cells, increase the blood supply to the brain, relieve the degree of cerebral edema and reduce intracranial pressure $(23,24)$. It has also been suggested that $33 \pm 1^{\circ} \mathrm{C}$ is the most significant temperature range for maintaining brain tissue function (25). In mice subjected to experimental cerebral ischemia with brain inflammation and mild hypothermia, Deng et al (26) determined that the content of ICAM-1 was significantly 
Table II. Changes in serum IL-1 $\beta$, IL-6, ICAM-1 and MMP-2 levels in the two groups ( $\mathrm{n}=40$ per group).

\begin{tabular}{|c|c|c|c|c|c|c|c|c|}
\hline \multirow[b]{2}{*}{ Group } & \multicolumn{2}{|c|}{$\mathrm{IL}-1 \beta(\mathrm{pg} / \mathrm{ml})$} & \multicolumn{2}{|c|}{ IL-6 (pg/ml) } & \multicolumn{2}{|c|}{ ICAM-1 $(\mu \mathrm{g} / \mathrm{ml})$} & \multicolumn{2}{|c|}{ MMP-2 $(\mu \mathrm{g} / \mathrm{l})$} \\
\hline & Before & $7 d$ & Before & $7 d$ & Before & $7 d$ & Before & $7 d$ \\
\hline $\mathrm{A}$ & $184.18 \pm 26.18$ & $138.57 \pm 15.48$ & $115.43 \pm 13.77$ & $58.77 \pm 5.46$ & $3.5 \pm 0.9$ & $3.0 \pm 0.5$ & $349.27 \pm 30.39$ & $219.12 \pm 26.33$ \\
\hline B & $186.20 \pm 22.23$ & $103.33 \pm 11.71$ & $117.44 \pm 9.13$ & $48.49 \pm 7.51$ & $3.4 \pm 0.8$ & $2.3 \pm 0.3$ & $352.19 \pm 32.51$ & $143.73 \pm 23.25$ \\
\hline P-value & 0.135 & 0.014 & 0.163 & 0.017 & 0.161 & 0.023 & 0.172 & 0.015 \\
\hline
\end{tabular}

Values are expressed as the mean \pm standard deviation. Before, prior to treatment; $7 \mathrm{~d}$, at 7 days after treatment was commenced; IL, interleukin; MMP, matrix metalloproteinase; ICAM, intercellular adhesion molecule.

Table III. Comparison of incidence of adverse reactions between the two groups ( $\mathrm{n}=40$ per group).

\begin{tabular}{lcccccc}
\hline & $\begin{array}{c}\text { Symptomatic } \\
\text { intracranial } \\
\text { hemorrhage }\end{array}$ & $\begin{array}{c}\text { Asymptomatic } \\
\text { intracranial } \\
\text { hemorrhage }\end{array}$ & $\begin{array}{c}\text { Extracranial } \\
\text { hemorrhage }\end{array}$ & $\begin{array}{c}\text { Pulmonary } \\
\text { infection }\end{array}$ & $\begin{array}{c}\text { Adverse } \\
\text { reaction rate (\%) }\end{array}$ & Mortalities \\
\hline A & 2 & 2 & 6 & 4 & 35 & $4(10)$ \\
B & 0 & 0 & 4 & 4 & 20 & $2(5)$ \\
P-value & & & & & 0.128 & 0.152 \\
\hline
\end{tabular}

Values are expressed as $\mathrm{n}$ or $\mathrm{n}(\%)$ unless otherwise specified.
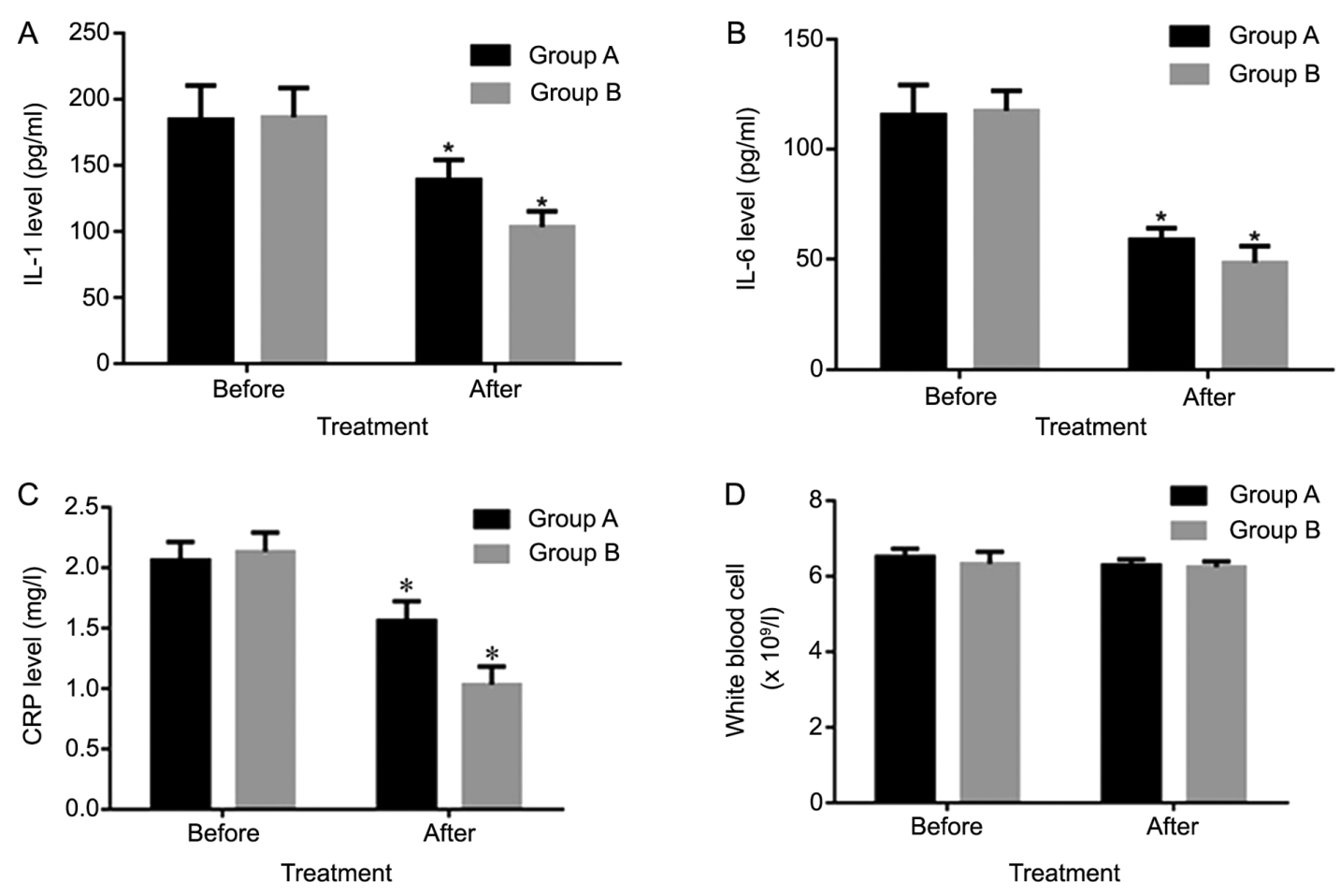

Figure 1. Changes in serum IL-1 $\beta$, IL-6 and CRP levels and the white blood cell count in the two groups. (A) There was no significant difference in serum IL-1 $\beta$ and IL-6 levels between the two groups prior to treatment $(\mathrm{P}>0.05)$. (B) At 7 days after treatment, group B and group A showed a significant decrease in serum IL-1 $\beta$ and IL-6 levels, the difference was statistically significant, $\mathrm{P}<0.05$. (C) CRP level before and after treatment in group A and B; (D) white blood cell number before and after treatment in group $\mathrm{A}$ and $\mathrm{B} .{ }^{*} \mathrm{P}<0.05$ compared with corresponding value prior to treatment. IL, interleukin; $\mathrm{CRP}, \mathrm{C}$-reactive protein.

lower than that in the control group, which indicated a certain inhibitory effect of mild hypothermia on brain inflammation. At the same time, associated animal experiments indicated that local hypothermia treatment may reduce the area of cerebral infarction $(27,28)$. With the continuous improvement of scientific research, an increasing number of studies 
Table IV. Correlation of inflammatory factors/cytokines with the National Institutes of Health Stroke Scale score.

\begin{tabular}{lccccr}
\hline Parameter & IL-1 $\beta$ & IL-6 & CRP & ICAM-1 & MMP-2 \\
\hline r & 0.461 & 0.547 & 0.612 & 0.367 & 0.436 \\
P-value & 0.021 & 0.019 & 0.006 & 0.031 & 0.029 \\
\hline
\end{tabular}

IL, interleukin; MMP, matrix metalloproteinase; ICAM, intercellular adhesion molecule; CRP, C-reactive protein.
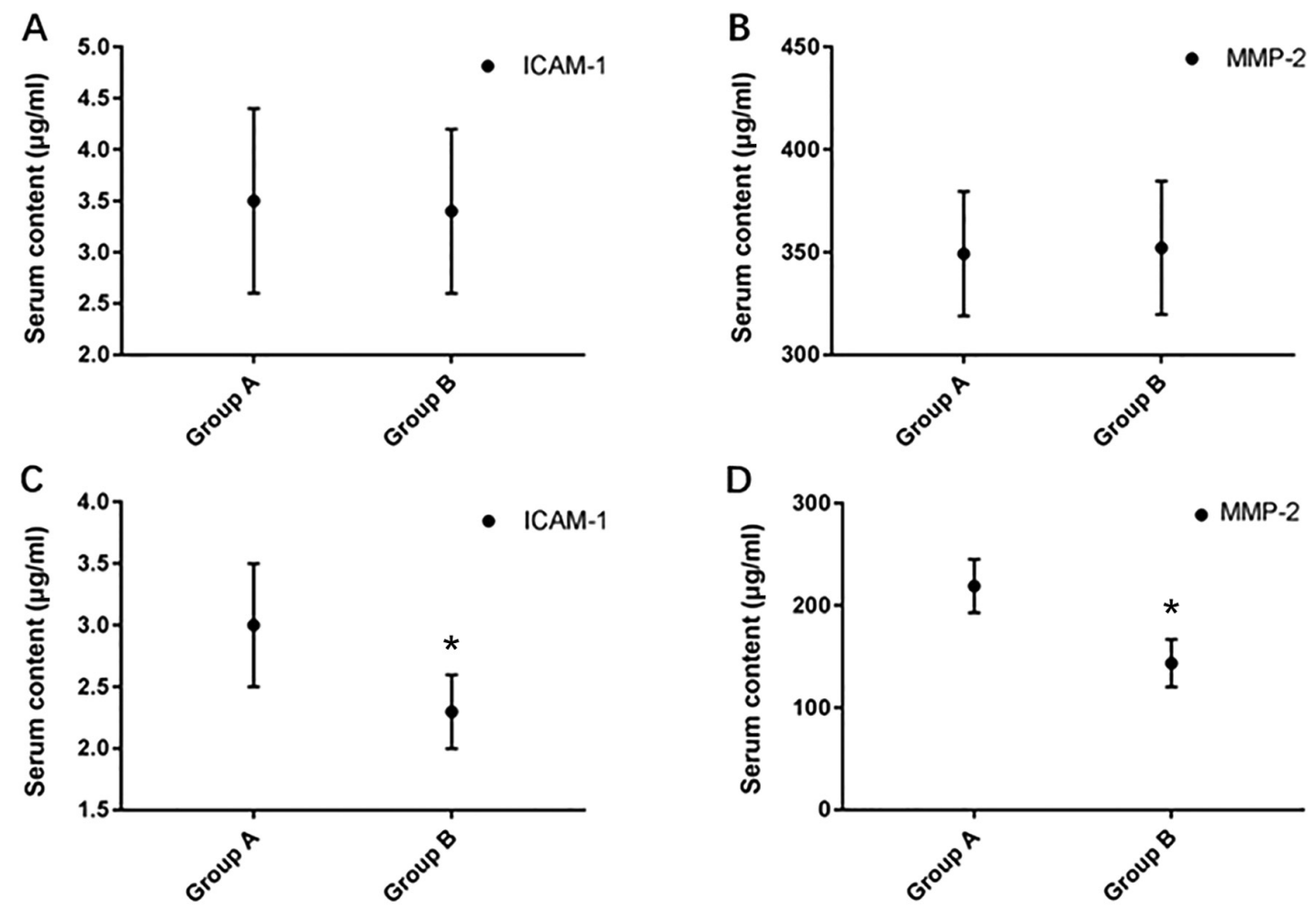

Figure 2. Differences in serum ICAM-1 and MMP-2 between the two groups. (A) ICAM-1 and (B) MMP-2 levels prior to treatment. (C) ICAM-1 and (D) MMP-2 levels at 7 days after treatment. ${ }^{*} \mathrm{P}<0.05$ vs. group A. MMP, matrix metalloproteinase; ICAM, intercellular adhesion molecule.

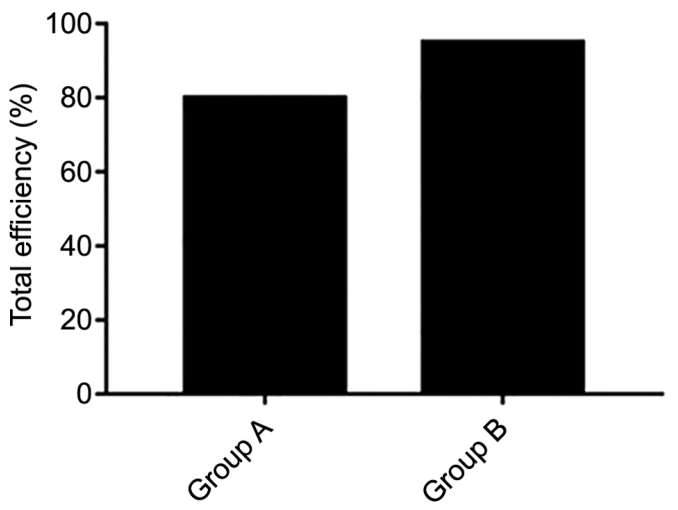

Figure 3. Evaluation of the effectiveness of the two treatments. The total effective rate in group B was significantly higher than that in group A $(\mathrm{P}<0.05)$.

have confirmed through experimental and clinical research that hypothermia treatment has a protective effect on neurological function, with good prognosis in the later stage and fewer side effects (29-31). Therefore, in the present clinical study, a treatment temperature of $33-35^{\circ} \mathrm{C}$ was adopted, which was compared with conventional treatment to determine the efficacy of intravenous thrombolysis combined with mild hypothermia treatment for acute cerebral infarction.

The results of the present study suggested that, compared with those in group A, the levels of IL-1 $\beta$, IL-6, CRP and ICAM-1 in group B were significantly decreased At 7 days after treatment $(\mathrm{P}<0.05)$. Compared with those prior to treatment, the levels of MMP-2 were significantly decreased At 7 days after treatment, and the levels of MMP-2 in group B was significantly lower than those in group $\mathrm{A}(\mathrm{P}<0.05)$. At 7 days after treatment, the total effective rate was $80 \%$ in group $\mathrm{A}$ and $95 \%$ in group B and there was a significant difference between the two groups $(\mathrm{P}<0.05)$. The incidence of adverse reactions in group A and group B was 35 and $20 \%$, respectively, and the mortality rates were 10 and $5 \%$, respectively. However, there were no significant differences in adverse events and mortalities between the two groups $(\mathrm{P}>0.05)$. In addition, a positive correlation of the level of IL-1 $\beta$, IL-6, CRP, ICAM-1 and MMP-2 with the NIHSS score was identified in the total cohort of patients prior to treatment. The above results suggest 
that, compared with intravenous thrombolytic therapy alone, intravenous thrombolysis combined with mild hypothermia treatment has the most obvious effects on the levels of IL-1 $\beta$, IL-6, CRP, ICAM-1 and MMP-2, and may significantly improve brain ischemia and hypoxia, reduce inflammation and ameliorate brain tissue damage.

In conclusion, intravenous thrombolysis combined with mild hypothermia treatment may significantly improve the clinical symptoms of patients with acute cerebral infarction and control the inflammatory response after cerebral infarction, indicating that this treatment is effective and may be used in the clinic. However, the exact mechanism of how the treatment affects the cytokines or markers in patients with acute cerebral infarction remains elusive. In the future, in vitro experiments are required to investigate the mechanisms of how this treatment alters the inflammatory cytokines.

\section{Acknowledgements}

Not applicable.

\section{Funding}

This study was supported by the Youth Medical Key Talent Project of Wuxi (grant no. QNRC084) and Health and Family Planning Commission of Wuxi (grant no. Q201610).

\section{Availability of data and materials}

All data generated or analyzed during this study are included in this published article.

\section{Authors' contributions}

CL and LHu contributed to conception and design and performed the experiments. JZ, MD, CF, XZ and LHan contributed to acquisition, analysis and interpretation of data. XZ, CL, JZ and LHan drafted the manuscript and revised it critically for important intellectual content and gave final approval of the version to be published. All authors approved revisions and the final paper, and agreed to be accountable for all aspects of the work in ensuring that questions related to the accuracy or integrity of any part of the work are appropriately investigated and resolved. All authors read and approved the final manuscript.

\section{Ethics approval and consent to participate}

All enrolled patients and their families provided written informed consent to participate in the present study and the study was approved by the Medical Ethics Committee of Wuxi Third People's Hospital (Wuxi, Jiangsu, China).

\section{Patient consent for publication}

All enrolled patients provided consent for the publication of this article.

\section{Competing interests}

The authors declare that they have no competing interests.

\section{References}

1. Zhang M: Clinical research on traumatic cerebral infarction treated with mild-hypothermia and acupuncture. Zhongguo Zhen Jiu 32: 697-700, 2012 (In Chinese).

2. Zhang X, Li Y, Liang Y, Sun P, Wu X, Song J, Sun X, Hong M, Gao P and Deng D: Distinguishing Intracerebral Hemorrhage from Acute Cerebral Infarction through Metabolomics. Rev Invest Clin 69: 319-328, 2017.

3. Muramatsu K, Fujino Y, Kubo T, Otani M, Fushimi K and Matsuda S: Efficacy of antimicrobial catheters for prevention of catheter-associated urinary tract infection in acute cerebral infarction. J Epidemiol 28: 54-58, 2017.

4. Juttler E, Kohrmann M and Schellinger PD: Therapy for early reperfusion after stroke. Nat Clin Pract Cardiovasc Med 3: 656-663, 2006

5. Varga V, Waje-Andreassen U, Naess H, Naess H, Lundstadsveen MT and Thomassen L: Thrombolytic therapy in cerebral infarction. Tidsskr Nor Laegeforen 129: 2214-2117, 2009 (In Norwegian).

6. Peng X, Yue W, Liu W, Dan B, Lin L and Tang Z: Protective roles of intra-arterial mild hypothermia and arterial thrombolysis in acute cerebral infarction. Springerplus 5: 1988, 2016.

7. Cechmanek BK, Tuor UI, Rushforth D and Barber PA: Very Mild hypothermia $\left(35^{\circ} \mathrm{C}\right)$ postischemia reduces infarct volume and blood/brain barrier breakdown following tPA Treatment in the Mouse. Ther Hypothermia Temp Manag 5: 203-208, 2015

8. Moon Y, Lee JH, Cho HJ, Roh HG, Choi JW, Chun YI and Kim HY: Intravenous thrombolysis in a patient with acute ischemic stroke attributable to intracranial dissection. Neurologist 18 : 136-138, 2012.

9. Miyauchi T, Wei EP and Povlishock JT: Evidence for the therapeutic efficacy of either mild hypothermia or oxygen radical scavengers after repetitive mild traumatic brain injury. J Neurotrauma 31: 773-781, 2014.

10. Guan Y, Zhao GD, Jiang L, et al: Meta-analysis of mild hypothermia in the treatment of acute ischemic stroke. J Practical Med 29: 1518-1521, 2013.

11. Chinese Medical Association Neurology Branch: Chinese Medical Association Neurology Branch Cerebrovascular Disease Group: Chinese guidelines for the diagnosis and treatment of acute ischemic stroke, 2014. Chin J Neurol 48: 246-257, 2015.

12. Hacke W, Kaste M, Fieschi C, Toni D, Lesaffre E, von Kummer R, Boysen G, Bluhmki E, Höxter G, Mahagne MH, et al: Intravenous thrombolysis with recombinant tissue plasminogen activator for acute hemispheric stroke. The European Cooperative Acute Stroke Study (ECASS). JAMA 274: 1017-1025, 1995.

13. Kruyt ND and Roos YWBM: Hyperglycemia in Acute Stroke: Humana Press, 2010.

14. Wu Y and Zhang X: Cost-effectiveness comparison of four drug regimens in the treatment of acute cerebral infarction. Chin $J$ Biochem Pharmaceutics 31: 57-58, 2010 (In Chinese).

15. Wang X, Shen B, Sun D and Cui X: Aspirin ameliorates cerebral infarction through regulation of TLR4/NF- $\kappa B$-mediated endoplasmic reticulum stress in mouse model. Mol Med Rep 17: 479-487, 2018.

16. Nakatani Y, Suto Y, Fukuma K, Yamawaki M, Sakata R, Takahashi S, Nakayasu H and Nakashima K: Intrathecal isoniazid for refractory tuberculous meningitis with cerebral infarction. Int Med 56: 953-957, 2017.

17. Fang ZQ: Experimental Methodology of Dialectical Treatment of Rats/Mice, Science Press, 2009.

18. Jiang Y and Lian YJ: Effects of Danhong injection on hemodynamics and the inflammation-related NF- $\mathrm{BB}$ signaling pathway in patients with acute cerebral infarction. Genet Mol Res 14: 16929-16937, 2015.

19. Yang $J$ and Yang Z: Changes of serum TNF- $\alpha$ sICAM-1 in patients with cerebral infarction and its clinical significance. J Brain Nervous Dis 10: 39-41, 2002 (In Chinese).

20. Kochanek PM and Hallenbeck JM: Polymorphonuclear leukocytes and monocytes/macrophages in the pathogenesis of cerebral ischemia and stroke 23: 1367-1379, 1992.

21. del Zoppo GJ, Schmidschönbein GW, Mori E, Copeland BR and Chang CM: Polymorphonuclear leukocytes occlude capillaries following middle cerebral artery occlusion and reperfusion in baboons. Stroke 22: 1276-1283, 1991. 
22. Yang $\mathrm{Y}, \mathrm{Wu} \mathrm{J}$ and Sui Z: Tumor necrosis factor and cerebral ischemia.Zhejiang J Integrated Trad Chin Western Med 19: 60-61, 2009 (In Chinese).

23. Hu Xiao, Qi Hao, Li Shirong, et al: The efficacy and possible mechanism of intravenous thrombolysis combined with mild hypothermia in patients with acute cerebral infarction. Chin J Neuropsychiatric Dis 42: 15-21, 2016 (In Chinese).

24. Gao HN, Lu HZ, Cao B, Du B, Shang H, Gan JH, Lu SH, Yang YD, Fang Q, Shen YZ, et al: Clinical findings in 111 cases of influenza A (H7N9) virus infection. N Engl J Med 368 : 2277-2285, 2013

25. Husain M: Avian influenza A (H7N9) virus infection in humans: epidemiology, evolution, and pathogenesis. Infect Genet Evol 28 304-312, 2014

26. Deng H, Han HS, Cheng D, Sun GH and Yenari MA: Mild hypothermia inhibits inflammation after experimental stroke and brain inflammation. Stroke 34: 2495-2501, 2003.
27. Chang YP, Liao PT, Shen EY and Liu IH: Protective effect against focal cerebral ischemia injury in acute phase of a novel invasive device for regional hypothermia. J Chin Med Assoc 78: 67-75, 2014.

28. Liang J: Effects of body temperature control on serum S-100B protein, LDH expression and prognosis in patients with severe head injury. Chin Laboratory Diagnosis 20: 755, 2016 (In Chinese).

29. Hu W, Dai Y, Chen S, et al: Clinical study of brain hypothermia on brain protection in patients with cerebral infarction. Chongqing Med J 45: 4817-4819, 2016 (In Chinese).

30. Andresen M, Gazmuri JT, Marín A, Regueira T and Rovegno M: Therapeutic hypothermia for acute brain injuries. Scand J Trauma Resusc Emerg Med 23: 42, 2015.

31. Guo XY, Li AQ, Zhan J, et al: Effects of local mild hypothermia on serum NSE in patients with severe cerebral infarction. Stroke and Neurological Diseases 19: 214-216, 2012. 